\title{
Neutrino oscillations refitted
}

\author{
D. V. Forero** M. Tórtola \\ AHEP Group, Institut de Física Corpuscular - C.S.I.C./Universitat de València, Parc Cientific de Paterna. \\ C/ Catedratico José Beltrán, 2 E-46980 Paterna (València) - SPAIN
}

\begin{abstract}
Here we update our previous global fit of neutrino oscillations by including the recent results which have appeared since the Neutrino-2012 conference. These include the measurements of reactor antineutrino disappearance reported by Daya Bay and RENO, together with latest T2K and MINOS data including both disappearance and appearance channels. We also include the revised results from the third solar phase of Super-Kamiokande, SK-III, as well as new solar results from the fourth phase of Super-Kamiokande, SK-IV. We find that the preferred global determination of the atmospheric angle $\theta_{23}$ is consistent with maximal mixing. We also determine the impact of the new data upon all the other neutrino oscillation parameters with emphasis on the increasing sensitivity to the CP phase, thanks to the interplay between accelerator and reactor data. In the appendix we present the updated results obtained after the inclusion of new reactor data presented at the Neutrino-2014 conference. We discuss their impact on the global neutrino analysis.
\end{abstract}

PACS numbers: 14.60.Pq, 13.15. $+\mathrm{g}, 26.65 .+\mathrm{t}, 12.15 . \mathrm{Ff}$

Keywords: Neutrino mass and mixing; neutrino oscillation; solar and atmospheric neutrinos; reactor and accelerator neutrinos

\section{INTRODUCTION}

The precise measurement of a non-zero value of the third mixing angle $\theta_{13}$ in the lepton mixing matrix [1 $[3]$ reported by the reactor experiments Daya Bay [4] and RENO [5] has played an important role in electroweak model-building as well as in the design of future upcoming experiments over the last two years. Compared to previous reactor anti-neutrino experiments CHOOZ [6] and Palo Verde [7], these new measurements have larger statistics, thanks to the increased reactor power and anti-neutrino detector size involved. More importantly, they have detectors located at different distances from the reactor cores, in order to reduce the effect of the systematic uncertainties, such as the ones associated to the predicted theoretical reactor fluxes. As a result these experiments have been able for the first time to observe the disappearance of reactor anti-neutrinos over short distances, of the order of $1 \mathrm{~km}$, providing the first robust measurement of the mixing angle $\theta_{13}$. Moreover, there have also been indications for a non-zero $\theta_{13}$ mixing angle coming from the observation of electron neutrino appearance on a muon neutrino beam at the accelerator oscillation experiments T2K [8] and MINOS [9].

Here we update the global fit of neutrino oscillations given in Ref. [10] by including the recent measurements of reactor anti-neutrino disappearance reported by the Daya Bay and RENO experiments [11 13], as well as accelerator appearance and disappearance results from MINOS and T2K [14 17]. Concerning the solar neutrino data, our analysis includes the recently revised results of the third solar phase of Super-Kamiokande, SK-III [18] as well as the latest results from its fourth solar phase, SK-IV, with lower energy threshold and improved systematic uncertainties [19]. We investigate the impact of the new data upon all the neutrino oscillation parameters, discussing in more detail the

\footnotetext{
*Electronic address: dvanegas@ific.uv.es

${ }^{\dagger}$ Electronic address: mariam@ific.uv.es

${ }^{\ddagger}$ Electronic address: valle@ific.uv.es
} 
status of the octant-determination of the atmospheric mixing angle, as well as the improved sensitivity to the CP phase $\delta$ that follows from the complementarity of accelerator and reactor neutrino data.

\section{UPDATED GLOBAL FIT MAY 2014}

\section{Updated solar neutrino analysis}

As in our previous global fit to neutrino oscillations 10 , here we consider the most recent results from the solar experiments Homestake [20], Gallex/GNO [21], SAGE [22], Borexino [23], SNO [24, 25] and the first three solar phases of Super-Kamiokande [18, 26, 27]. Here we have included the revised results from the third solar phase of Super-Kamiokande, published in December 2012 in the arXiv version of Ref. [18]. This revision corrects the estimated systematic error on the total flux observed in Super-Kamiokande as well as the total ${ }^{8} \mathrm{~B}$ flux calculation. We find that the changes are very small and their impact on the determination of solar oscillation parameters is hardly noticeable. We also include the results from the fourth solar phase of Super-Kamiokande, SK-IV [19]. This data release corresponds to 1306.3 live-days and is presented in the form of 23 day and night energy bins. Thanks to several improvements in the hardware and software of Super-Kamiokande, an improved systematic uncertainty as well as a very low energy threshold of 3.5 MeV have been achieved. As we will discuss later, these new data consolidate the previous Super-Kamiokande solar data releases, with a minor impact in the global fit to neutrino oscillations. More detailed information on our simulation and analysis of solar neutrino data can be found in Refs. [10, 28, 29].

\section{New reactor data}

For the statistical analysis of reactor data we follow the same strategy as in our previous paper [10]. We define a $\chi^{2}$ that compares the observed and measured event rates at each anti-neutrino detector. Several pull parameters are introduced in order to account for the different systematical errors associated to the reactor, detector and background uncertainties. An absolute normalization factor is left free in the fit, to be determined from the experimental data. This technique is also used in the official analyses performed by the Daya Bay and RENO Collaborations [5, 30]. For the analysis of reactor data we take into account the total rate analysis of the latest Double Chooz data in Ref. 31 , already discussed in our previous fit, as well as the new reactor data released by Daya Bay and RENO and described below.

\section{Daya Bay}

Daya Bay is a reactor experiment with six anti-neutrino detectors, arranged in three experimental halls (EHs). Two detectors, located in EH1, one in EH2 and three in EH3. EH1 and EH2 are considered as near detectors, while EH3 is the far detector. Electron anti-neutrinos are generated in six reactor cores, distributed in pairs, with equal thermal power $\left(\mathrm{P}_{\mathrm{th}}^{\mathrm{r}}=2.9 \mathrm{GW}_{\mathrm{th}}\right)$ and detected in the EHs. The effective baselines are $512 \mathrm{~m}$ and $561 \mathrm{~m}$ for the near halls and $1579 \mathrm{~m}$ for the far [11]. With baseline $\sim \mathrm{km}$ Daya Bay is sensitive to the first dip in the $\bar{\nu}_{e}$ disappearance probability. Using this near-far technique Daya Bay has minimized the systematic errors thus providing the most precise determination of the reactor mixing angle $\theta_{13}$. In Refs. [11, 12] Daya Bay reported 217 days of data collected. Such high statistics sample leads to a substantial improvement in the statistical errors compared to the previous analysis in Ref. [30]. Using the corresponding event rates at the six anti-neutrino detectors we obtain an improved measurement of the reactor mixing angle. 
RENO

The Reactor Experiment for Neutrino Oscillations (RENO) is a short baseline reactor neutrino oscillation experiment located in South Korea. RENO consists of six reactor cores with maximum powers ranging from $2.66 \mathrm{GW}_{\text {th }}$ to $2.8 \mathrm{GW}_{\text {th }}$ and two identical anti-neutrino detectors located at 294 and $1383 \mathrm{~m}$ from the center of the reactor array. With both near and far detectors, RENO provided an important confirmation of the first Daya Bay measurement of $\theta_{13}$ [5]. We use their updated results presented at the TAUP 2013 conference [13], consisting of 403 days of data-taking, with improved systematic uncertainties, background estimates and energy calibration.

\section{New long-baseline neutrino data}

Over the last two years new data on $\nu_{\mu}$ disappearance and $\nu_{e}$ appearance have been released by the long-baseline (LBL) accelerator experiments MINOS and T2K. Below we summarize the most recent data from both experiments included in our global fit. As in our previous analysis, we use the GLOBES software package 32] for the simulation and statistical analysis of accelerator neutrino oscillation data from MINOS and T2K. The expected event numbers for a given channel in a particular detector are determined using the full three-neutrino survival probability with the relevant matter effects. As we will see, these data will play an important role in the global fit, since they provide key contributions to the determination of the atmospheric oscillation parameters and the CP violation phase. We now discuss them separately.

\section{Disappearance channel in MINOS}

The latest measurements of the $\nu_{\mu}$ disappearance channel in MINOS have been published in Ref. [14]. These results come from the full MINOS data set, collected over a period of nine years and correspond to exposures of $10.71 \times 10^{20}$ protons on target (POT) in the $\nu_{\mu}$-dominated beam and $3.36 \times 10^{20}$ POT in the $\bar{\nu}_{\mu}$-enhanced beam. One of the key features of these data sample is the preference for a non-maximal value of the atmospheric mixing angle $\theta_{23}$. In fact, from the official MINOS analysis, one obtains that maximal mixing is disfavoured at the $86 \%$ C.L.

\section{Appearance channel in MINOS}

The most recent results for the searches of $\nu_{e}$ appearance in MINOS have been reported in Ref. [15]. These data correspond to exposures of $10.6 \times 10^{20}$ POT in the neutrino channel and $3.3 \times 10^{20}$ POT in the anti-neutrino channel. The neutrino sample is the same as in the preliminary results presented in the Neutrino-2012 conference, used in our previous analysis. However, there are some differences in the reconstructed energy distributions. We are now using the full update from Ref. [15].

\section{Disappearance channel in T2K}

The latest results for the $\nu_{\mu}$ disappearance channel in T2K have been collected from January 2010 to May 2013, during the four runs of the experiment and correspond to a total exposure of $6.57 \times 10^{20} \mathrm{POT}$ [16]. In comparison with the previous $\mathrm{T} 2 \mathrm{~K}$ results in Ref. [33], sensitivities have been improved thanks to new event selection and reconstruction techniques, as well as higher statistics at the near off-axis detector. A total number of 120 muon neutrino event candidates have been observed at the far detector while $446.0 \pm 22.5$ (sys.) events were expected in 
absence of oscillations. As we will see in the next section, the T2K disappearance data now provides the most precise measurement for the atmospheric mixing angle $\theta_{23}$ with better sensitivity than all other experiments. Moreover, in contrast to the MINOS $\nu_{\mu}$ disappearance data, they prefer a best fit $\theta_{23}$ value very close to maximal. This point will be crucial for the $\theta_{23}$ octant (in)determination from the global neutrino oscillation analysis.

Appearance channel in T2K

As for the disappearance channel, the latest available T2K appearance data correspond to a total exposure of $6.57 \times 10^{20}$ POT, collected from run 1 to run 4 in the experiment [17]. A total of $4.92 \pm 0.55$ background events were expected in the absence of oscillations, while a sample of 28 electron neutrino events have been detected. The observed event distribution is consistent with an appearance signal at $7.3 \sigma$.

\section{GLOBAL FIT 2014 RESULTS}

In addition to the solar, reactor and long-baseline accelerator neutrino data described in the previous section, in our global fit to neutrino oscillations we also include the last results from the KamLAND reactor experiment presented in Ref. [34] as well as the atmospheric neutrino analysis provided by the Super-Kamiokande Collaboration in Ref. [35].

\section{A. The role of long-baseline neutrino data in atmospheric parameter determination}

Long-baseline neutrino data have by now achieved very good precision. In fact the determination of the atmospheric oscillation parameters has become fully dominated by the combination of T2K and MINOS data. This can be appreciated from Fig. 1. where one sees how the latest T2K data places the best constraint on the atmospheric angle $\theta_{23}$, while MINOS still provides the best determination for the atmospheric mass splitting $\Delta m_{31}^{2}$. Atmospheric neutrino data from Super-Kamiokande are in full agreement with the parameter regions determined by long-baseline results, though with less sensitivity. In this figure we confirm the result obtained by the experimental collaborations about the maximality of the atmospheric angle. MINOS data have a mild preference for non maximal $\theta_{23}$, although $\theta_{23}=\pi / 4$ is inside the $90 \%$ CL region for 2 d.o.f. The absolute best fit point from the analysis of MINOS lies in the first octant, $\theta_{23}<\pi / 4$, although values in the second octant are allowed with very small $\Delta \chi^{2}$. Concerning T2K data, one sees that both for normal (left panel) and inverted mass hierarchy (right panel) the best fit value is very close to maximal: $\sin ^{2} \theta_{23}=0.52$ in both cases, maximal mixing being allowed with very small $\Delta \chi^{2}$ with respect to the absolute minimum: 0.03 (0.02) for normal (inverted) mass ordering. The global fit preference for values of $\theta_{23}$ in the second octant emerges after the combination with reactor data, as we will discuss in the next subsection. We find the best fit points:

$$
\begin{array}{ll}
\sin ^{2} \theta_{23}=0.567_{-0.128}^{+0.032} & \Delta m_{31}^{2}=\left(2.48_{-0.07}^{+0.05}\right) \times 10^{-3} \mathrm{eV}^{2} \quad \text { (normal hierarchy) } \\
\sin ^{2} \theta_{23}=0.573_{-0.043}^{+0.025} & \Delta m_{31}^{2}=\left(2.38_{-0.06}^{+0.05}\right) \times 10^{-3} \mathrm{eV}^{2} \quad \text { (inverted hierarchy) }
\end{array}
$$

Note that for normal hierarchy $(\mathrm{NH})$ a local minimum appears in the first octant $\left(\sin ^{2} \theta_{23}=0.467\right)$ with $\Delta \chi^{2}=0.28$ with respect to the global minimum. For the case of inverted hierarchy (IH), solutions with $\sin ^{2} \theta_{23} \leq 0.5$ appear only with $\Delta \chi^{2}>1.7$. Comparing with our previous global fit, we see that best fit values for the atmospheric mixing angle are slightly shifted towards maximal values thanks to the latest T2K data. Likewise, $\Delta m_{31}^{2}$ values are also shifted towards lower values due to T2K data, which now prefer smaller values of the atmospheric mass splitting. 

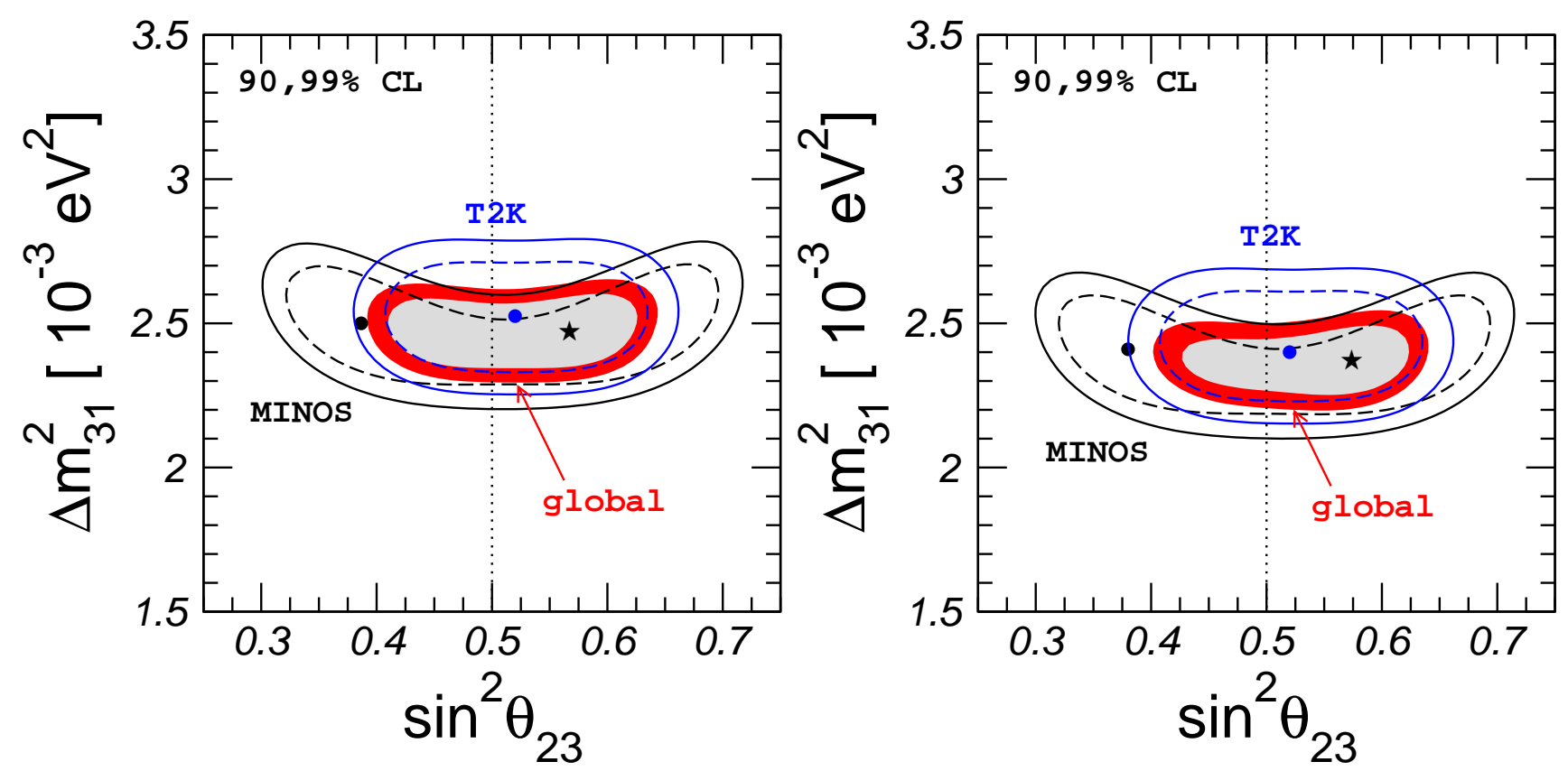

FIG. 1: 90 and 99\% C.L. regions in the $\sin ^{2} \theta_{23}-\Delta m_{31}^{2}$ plane from separate analysis of MINOS (black lines), T2K (blue lines) and from the global analysis of all data samples (coloured regions). The left (right) panel corresponds to normal (inverted) mass ordering.

\section{B. The $\theta_{23}$ octant and the CP violation phase $\delta$}

In this section we will discuss the complementarity between long-baseline accelerator and reactor neutrino data in the determination of the $\theta_{23}$ octant as well as the CP phase $\delta$. We will quantify the new sensitivity in the CP violation phase $\delta$ as well as the octant of the atmospheric mixing angle $\theta_{23}$. This emerges by combining the latest accelerator with the latest reactor data.

We start by discussing the effect of the different data samples upon the possible preference for a given octant of $\theta_{23}$. Our results are shown in Fig. 2. There we display the allowed regions at $\Delta \chi^{2}=1,4,9$ in the $\sin ^{2} \theta_{23}-\sin ^{2} \theta_{13}$ plane for normal (upper panels) and inverted (lower panels) neutrino mass hierarchy. In order to appreciate the effect of the individual data sample combinations on the parameter determinations we have prepared three different panels in this plane. The left panel is obtained by the combination of the long-baseline data from MINOS and T2K and the results of all solar neutrino experiments plus KamLAND. The accelerator MINOS and T2K data already produce a rather restricted allowed region in parameter space, showing an anti-correlation between $\theta_{23}$ and $\theta_{13}$ coming essentially from the oscillation probability in the $\nu_{e}$ appearance channel. In this panel solar and KamLAND impose only minor constraints on the reactor mixing angle $\theta_{13}$. In the middle panel of Fig. 22 the data samples from Double Chooz, Daya Bay and RENO have been included in the analysis. Here one can see how the very precise determination of $\theta_{13}$ at reactor experiments, particularly Daya Bay, considerably reduces the allowed region. On the other hand, the Daya Bay preference for values of $\sin ^{2} \theta_{13}$ around $0.023-0.024$ moves the best fit value of $\theta_{23}$ to the second octant. This effect is particularly important for the case of inverted hierarchy, because of the slightly larger values of $\theta_{13}$ preferred for $\theta_{23}<\pi / 4$. As a result, the first octant region is more strongly disfavoured so that values of $\sin ^{2} \theta_{23}<\pi / 4$ are allowed only with $\Delta \chi^{2}>1.5$. Finally, the right-most panel shows the allowed regions after the inclusion of the Super-Kamiokande atmospheric data [35]. One can see that there is basically no change between middle and right panel. This follows from the fact that the analysis of atmospheric data we adopt does not show a particular preference for any octant of $\theta_{23}$, both of which are allowed at $1 \sigma$. This behaviour is also confirmed in the preliminary versions 


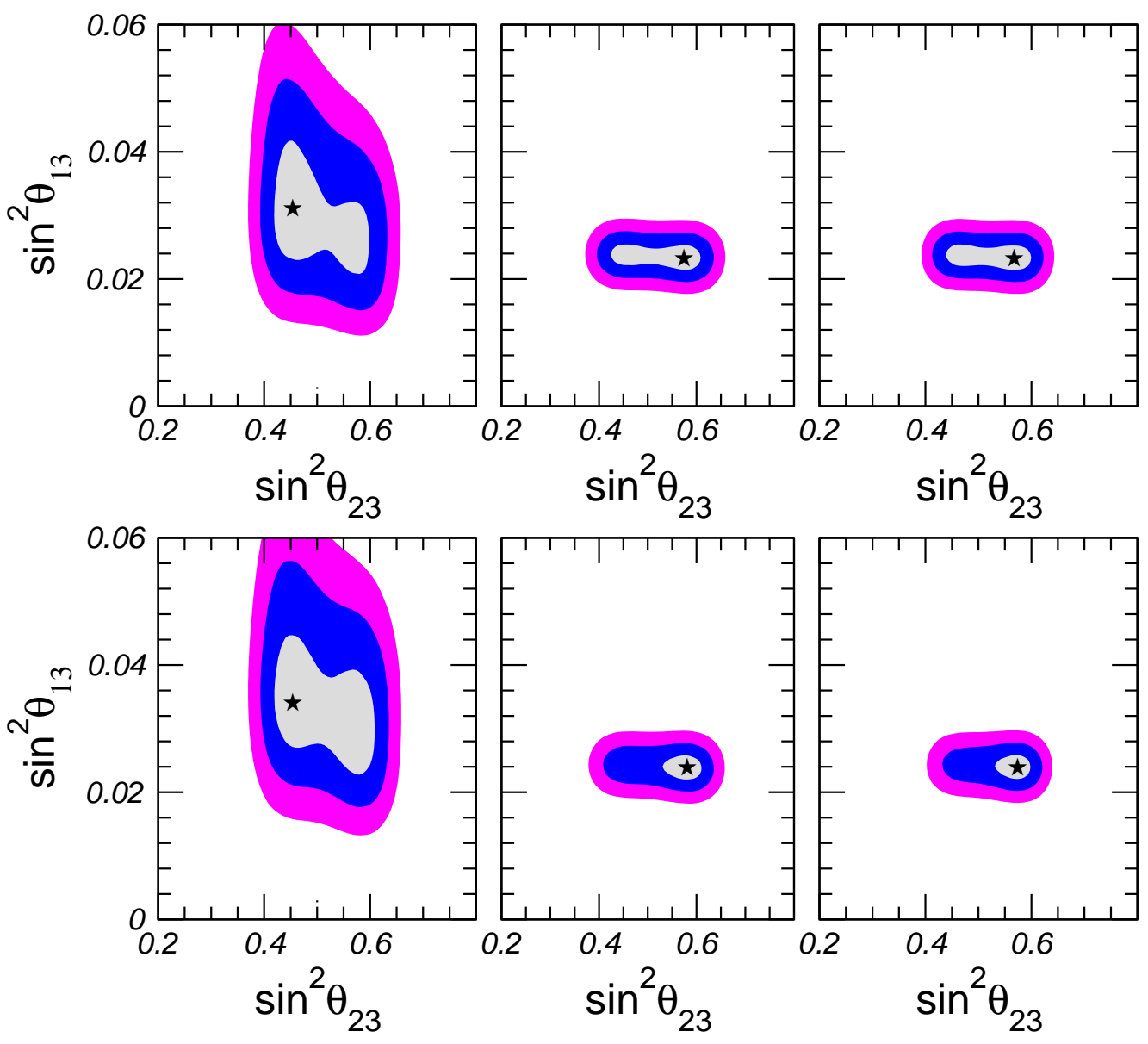

FIG. 2: Upper panels: contour regions with $\Delta \chi^{2}=1,4,9$ in the $\sin ^{2} \theta_{23}-\sin ^{2} \theta_{13}$ plane from the analysis of long-baseline $($ MINOS and T2K) + solar + KamLAND data (left panel), long-baseline + solar + KamLAND + new Double Chooz, Daya Bay and RENO reactor data (middle panel) and the global combination (right panel) for normal hierarchy. Lower panels, the same but for inverted neutrino mass hierarchy.

of updated Super-Kamiokande analysis in Refs. 36, 37.

Now we turn to the discussion of the sensitivity to the $\mathrm{CP}$ violation phase, $\delta$. Our previous global analysis in Ref. [10] showed essentially no dependence on this phase. However the new results on $\nu_{e}$ appearance at long-baseline experiments in combination with the very precise measurement of $\theta_{13}$ at reactor experiments provides, for the first time, a substantial sensitivity to the $\mathrm{CP}$ phase $\delta$. This new effect is illustrated in Fig. 3 . Here, left panels show the allowed regions with $\Delta \chi^{2}=1,4,9$ in the $\sin ^{2} \theta_{13}-\delta$ plane from the analysis of long-baseline accelerator data from MINOS and T2K, in both appearance as well as disappearance channels. This is indicated by three different line styles used in the left panels. On the other hand, the coloured regions correspond to the results obtained from the global oscillation -analysis. As expected the combination with reactor data results in narrower regions for $\theta_{13}$. One can also notice that there is a mismatch between the region of $\theta_{13}$ preferred by accelerator data for values of the CP phase $\delta$ around $0.5 \pi$ and the measured value of this mixing angle at reactor experiments such as Daya Bay, which dominates the best fit determination. As a result of this mismatch one obtains in the global analysis a significant rejection for values of $\delta$ phase around $0.5 \pi$. This can be seen in the right panels of Fig. 3 Here one notices that for normal hierarchy values of $\delta \simeq \pi / 2$ are disfavoured with $\Delta \chi^{2}=3.4(1.8 \sigma)$, while for inverted hierarchy they are disfavoured with $\Delta \chi^{2}=6.2(2.5 \sigma)$. In both cases the preferred $\delta$ value is located close to $1.5 \pi$. The best fit points 

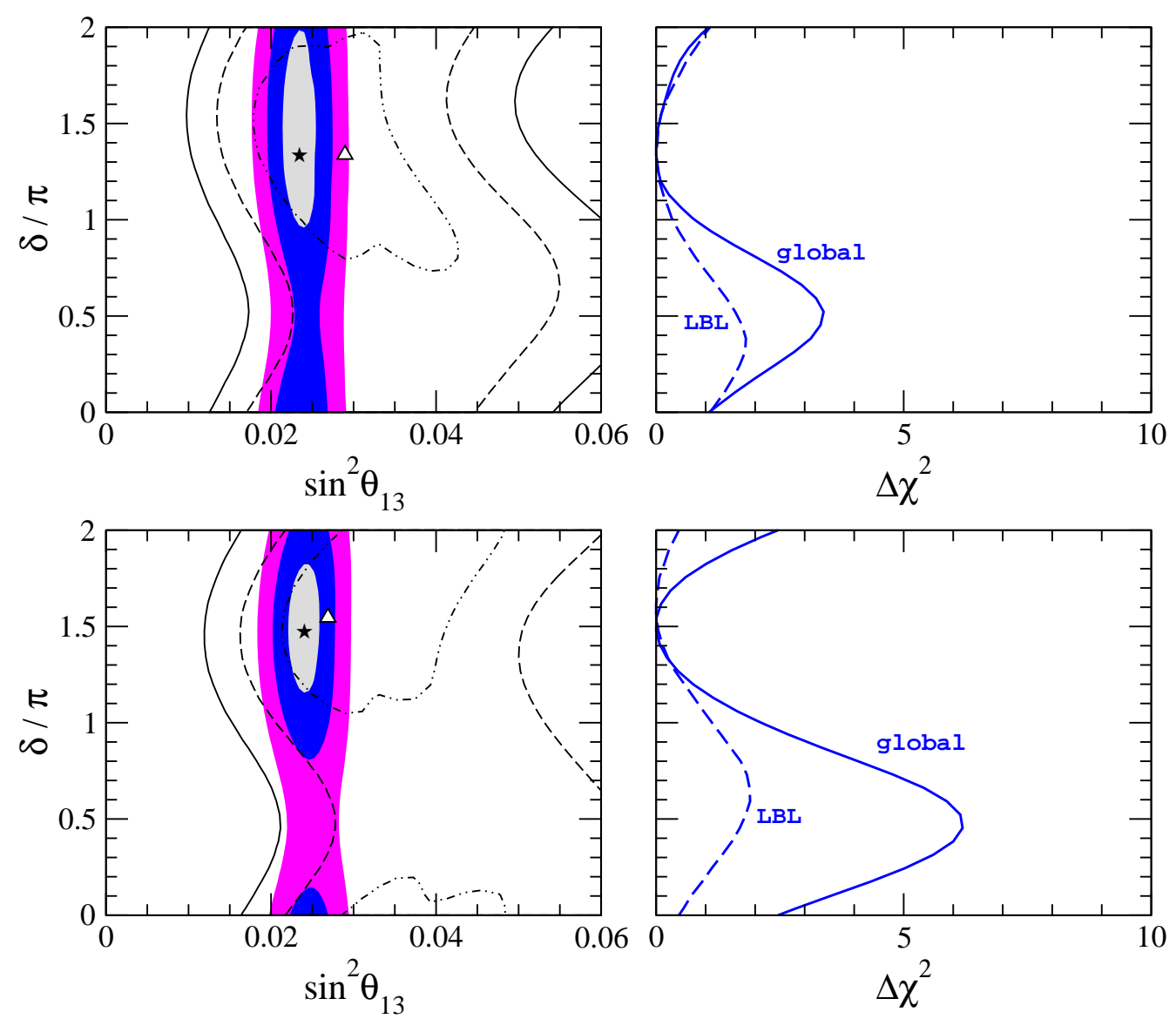

FIG. 3: Left panels: contour regions with $\Delta \chi^{2}=1,4,9$ in the $\theta_{13}-\delta$ plane from the analysis of LBL data alone (lines) and from the combined global analysis (coloured regions). Right panels: $\Delta \chi^{2}$ as a function of the CP-violating phase $\delta$ from the analysis of LBL data (dashed line) as well as from the global analysis (solid line). Upper (lower) figures correspond to NH (IH).

and $1 \sigma$ errors on $\delta$ are given by:

$$
\begin{array}{ll}
\delta=\left(1.34_{-0.38}^{+0.64}\right) \pi & \text { (normal hierarchy) } \\
\delta=\left(1.48_{-0.32}^{+0.34}\right) \pi & \text { (inverted hierarchy) }
\end{array}
$$

Comparing now with other global neutrino oscillation analyses in the literature we find our results on the CP phase qualitatively agree with the ones in Refs. [38, 39] for the same data included. Note, however, that the agreement holds for their global analysis without atmospheric data, since these authors have also included the effect of the $\delta$ in the atmospheric data sample, absent in the official Super-Kamiokande analysis adopted here. As a result, their global fit results show a somewhat stronger rejection against $\delta \simeq \pi / 2$ than we find, specially for the normal hierarchy case, as expected. In the inverted hierarchy case, though, the sensitivity on the CP phase is essentially unaffected by the subleading effects on the atmospheric analysis.

\section{Summary of global fit}

In this section we summarize the results obtained in our global analysis to neutrino oscillations. In Fig. 4 we present the $\Delta \chi^{2}$ profiles as a function of all neutrino oscillation parameters. In the panels with two lines, the solid one corresponds to normal hierarchy while the dashed one gives the result for inverted mass hierarchy. Best fit values 

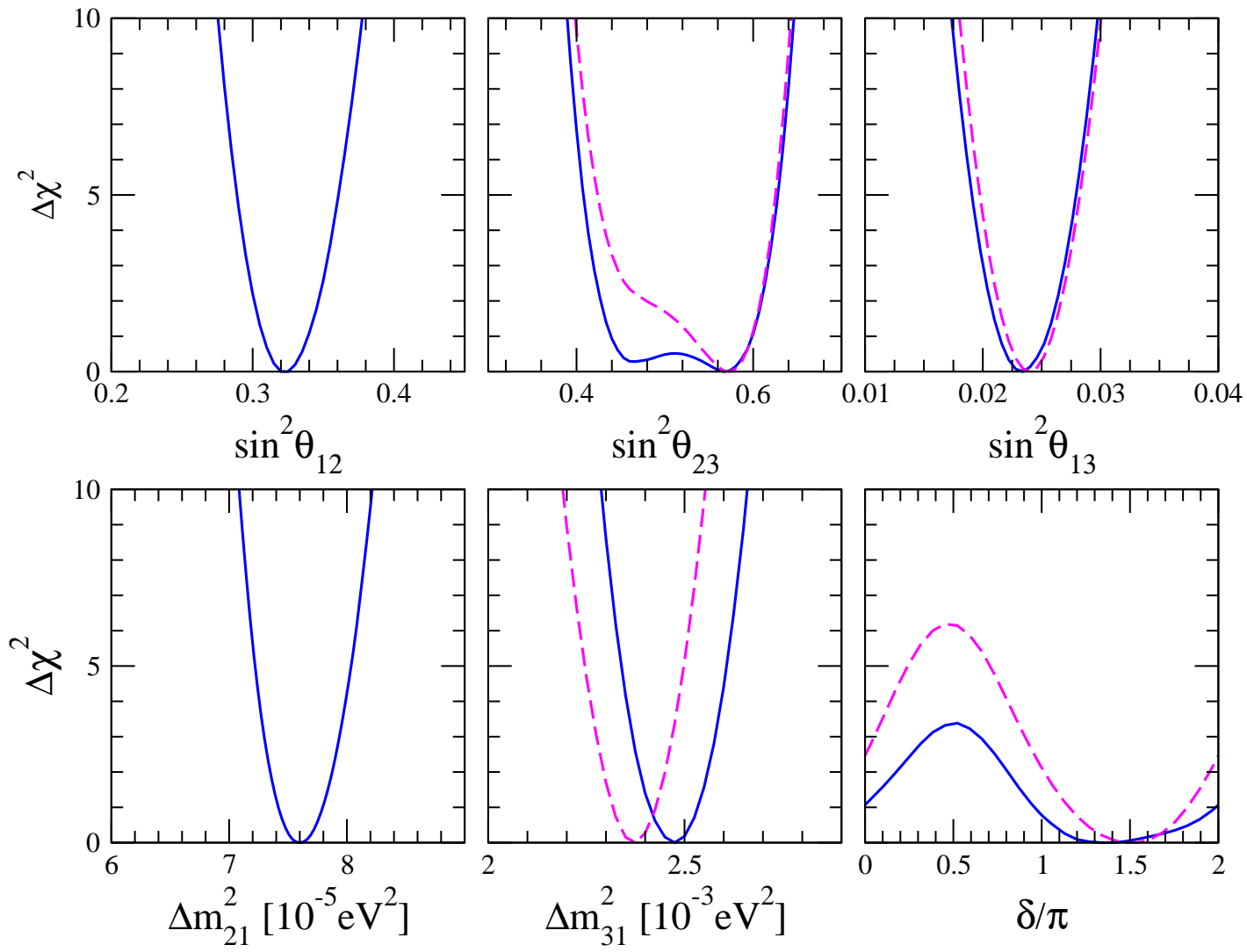

FIG. 4: $\Delta \chi^{2}$ profiles as a function of all the neutrino oscillation parameters $\sin ^{2} \theta_{12}, \sin ^{2} \theta_{23}, \sin ^{2} \theta_{13}, \Delta m_{21}^{2}, \Delta m_{31}^{2}$ and the $\mathrm{CP}$ phase $\delta$. For the central and right panels the solid lines correspond to the case of normal mass hierarchy while the dashed ones correspond to inverted mass hierarchy.

as well as 1,2 and $3 \sigma$ allowed ranges for all the neutrino oscillation parameters are reported in Table \.

First we note that solar neutrino parameter determination is basically unchanged with respect to our previous global fit in [10]. We find that the inclusion of the new SK-IV solar data sample leads only to minor modifications in the $\sin ^{2} \theta_{12}$ and $\Delta m_{21}^{2}$ best fit values. As we already discussed in the previous section, the atmospheric neutrino parameters are now determined mainly by the new long-baseline data. With the new T2K data, the preferred value for the mass splitting $\Delta m_{31}^{2}$ is now somewhat smaller, while the best fit value for the atmospheric angle $\theta_{23}$ has been shifted towards values closer to maximal. The status of maximal $\theta_{23}$ mixing angles has also been improved thanks to the latest $\mathrm{T} 2 \mathrm{~K}$ disappearance data. Regarding the reactor mixing angle $\sin ^{2} \theta_{13}$, the more precise reactor data from Daya Bay and RENO have reduced the allowed $1 \sigma$ range from $\sim 11 \%$ to $\sim 8 \%$. The preferred value of $\theta_{13}$ has also been shifted to somewhat smaller values. Finally, thanks to the combination of the latest accelerator and reactor neutrino data, we have obtained an enhanced sensitivity to the $\mathrm{CP}$ violation phase. We find preferred values for $\delta$ around $1.5 \pi$ for both mass hierarchies. On the other hand, values close to $0.5 \pi$ are disfavoured at $1.8 \sigma(2.5 \sigma)$ for normal (inverted) mass ordering.

\section{CONCLUSIONS}

Here we have updated the global fit of neutrino oscillations given in Ref. [10] by including the recent measurements of the last two years. These include the measurements of reactor anti-neutrino disappearance reported by Daya Bay and RENO, together with latest long-baseline appearance and disappearance data from T2K and MINOS. In 


\begin{tabular}{lccc}
\hline parameter & best fit $\pm 1 \sigma$ & $2 \sigma$ range & $3 \sigma$ range \\
\hline$\Delta m_{21}^{2}\left[10^{-5} \mathrm{eV}^{2}\right]$ & $7.60_{-0.18}^{+0.19}$ & $7.26-7.99$ & $7.11-8.18$ \\
$\left|\Delta m_{31}^{2}\right|\left[10^{-3} \mathrm{eV}^{2}\right](\mathrm{NH})$ & $2.48_{-0.07}^{+0.05}$ & $2.35-2.59$ & $2.30-2.65$ \\
$\left|\Delta m_{31}^{2}\right|\left[10^{-3} \mathrm{eV}^{2}\right](\mathrm{IH})$ & $2.38_{-0.06}^{+0.05}$ & $2.26-2.48$ & $2.20-2.54$ \\
$\sin ^{2} \theta_{12} / 10^{-1}$ & $3.23 \pm 0.16$ & $2.92-3.57$ & $2.78-3.75$ \\
$\theta_{12} /^{\circ}$ & $34.6 \pm 1.0$ & $32.7-36.7$ & $31.8-37.8$ \\
$\sin ^{2} \theta_{23} / 10^{-1}(\mathrm{NH})$ & $5.67_{-1.28}^{+0.32}$ & & \\
$\theta_{23} /^{\circ}$ & $48.9_{-7.4}^{+1.9}$ & $40.0-52.1$ & $38.8-53.3$ \\
$\sin ^{2} \theta_{23} / 10^{-1}(\mathrm{IH})$ & $5.73_{-0.43}^{+0.25}$ & $4.32-6.21$ & $4.03-6.40$ \\
$\theta_{23} /^{\circ}$ & $49.2_{-2.5}^{+1.5}$ & $41.1-52.0$ & $39.4-53.1$ \\
$\sin ^{2} \theta_{13} / 10^{-2}(\mathrm{NH})$ & $2.34 \pm 0.20$ & $1.95-2.74$ & $1.77-2.94$ \\
$\theta_{13} /^{\circ}$ & $8.8 \pm 0.4$ & $8.0-9.5$ & $7.7-9.9$ \\
$\sin ^{2} \theta_{13} / 10^{-2}(\mathrm{IH})$ & $2.40 \pm 0.19$ & $2.02-2.78$ & $1.83-2.97$ \\
$\theta_{13} /^{\circ}$ & $8.9 \pm 0.4$ & $8.2-9.6$ & $7.8-9.9$ \\
$\delta / \pi(\mathrm{NH})$ & $1.34_{-0.38}^{+0.64}$ & $0.0-2.0$ & $0.0-2.0$ \\
$\delta /{ }^{\circ}$ & $241_{-68}^{+115}$ & $0-360$ & $0-360$ \\
$\delta / \pi(\mathrm{IH})$ & $1.48_{-0.32}^{+0.34}$ & $0.0-0.14 \& 0.81-2.0$ & $0.0-2.0$ \\
$\delta /{ }^{\circ}$ & $266_{-58}^{+61}$ & $0-25 \& 146-360$ & $0-360$ \\
\hline
\end{tabular}

${ }^{a}$ There is a local minimum in the first octant, $\sin ^{2} \theta_{23}=0.467$ with $\Delta \chi^{2}=0.28$ with respect to the global minimum

TABLE I: Neutrino oscillation parameters summary. For $\Delta m_{31}^{2}, \sin ^{2} \theta_{23}, \sin ^{2} \theta_{13}$, and $\delta$ the upper (lower) row corresponds to normal (inverted) neutrino mass hierarchy.

addition, we have also included the revised data form the third solar phase of Super-Kamiokande, SK-III, as well as new solar results from the fourth phase of Super-Kamiokande, SK-IV. Our results are summarized in the four figures and Table. We find that for normal mass ordering the global best fit value of the atmospheric angle $\theta_{23}$ is consistent with maximal mixing at one-sigma, while for the inverted spectrum maximal mixing appears at $1.3 \sigma$. We note that the T2K disappearance data now provide the most sensitive measurement of the atmospheric mixing angle $\theta_{23}$. We also determine the impact of the new data upon all the other neutrino oscillation parameters, with emphasis on the increasing sensitivity to the $\mathrm{CP}$ violation phase $\delta$. The latter follows from the complementarity between accelerator and reactor data and leads to preferred ranges given in the table.

\section{Acknowledgments}

This work was supported by the Spanish MINECO under grants FPA2011-22975 and MULTIDARK CSD2009-00064 (Consolider-Ingenio 2010 Programme).

\section{APPENDIX: Updated global analysis after Neutrino 2014 conference}

In this Appendix we present an updated global fit after the inclusion of new data released at the Neutrino 2014 conference in Boston in June 2014. We have included the latest data from the reactor experiments Double Chooz, RENO, and Daya Bay. 
Double Chooz presented new results corresponding to 467.9 live days [40, 41]. The analysis of new data, with twice more statistics than the previous release, improved energy reconstruction, and lower systematics, implies a slight improvement in the determination of the reactor mixing angle from the rate + shape analysis:

$$
\sin ^{2} 2 \theta_{13}(\text { Double Chooz })=0.090_{-0.029}^{+0.032}
$$

The RENO experiment released their new rate-only analysis using 800 days of data taking [42].

$$
\sin ^{2} 2 \theta_{13}(\mathrm{RENO})=0.101 \pm 0.008(\text { stat }) \pm 0.010(\text { syst })
$$

also improving their former determination of $\theta_{13}$.

Finally, the Daya Bay Collaboration presented their results for a period of 621 days (four times more statistics than their previous data release), combining the periods with six and eight antineutrino detectors [43]. From their rate + shape analysis, they obtain the following best fit value:

$$
\sin ^{2} 2 \theta_{13}(\text { Daya Bay })=0.084 \pm 0.005
$$

now determined with an improved $6 \%$ precision, and slightly lower compared to the previous value.

A distortion in the reactor spectrum in the energy range between 4 and $6 \mathrm{MeV}$ was reported by the RENO and Double Chooz collaborations at the Neutrino 2014 conference [41, 42]. The origin of this effect, also confirmed by Daya Bay [44], is still unknown, although its correlation with the reactor thermal power indicates it may be consistent with an unaccounted reactor neutrino flux. In any case, this excess of events around $5 \mathrm{MeV}$ does not affect the determination of $\theta_{13}$ from reactor experiments, based upon the comparison of near and far detector rates.

\section{A. Impact of new reactor data upon the global oscillation fit}

The main difference between this update and the analysis in the previous sections is a slightly lower and more precise value of $\theta_{13}$ implied by the recent Daya Bay reactor data:

$$
\begin{aligned}
& \sin ^{2} \theta_{13}=0.0226 \pm 0.0012(\mathrm{NH}) \\
& \sin ^{2} \theta_{13}=0.0229 \pm 0.0012(\mathrm{IH}) .
\end{aligned}
$$

This result has an impact upon the determination of $\sin ^{2} \theta_{23}$ through the correlations between these two mixing angles. In particular, the slightly lower value of $\sin ^{2} \theta_{13}$ preferred by the new Daya Bay data favors values of $\theta_{23}$ in the second octant, worsening the status of the first octant solution. Comparing with the situation before the Neutrino 2014 conference, we find that for $\mathrm{NH}$ the local minimum in the first octant appears now with $\Delta \chi^{2}=0.36$ (vs $\Delta \chi^{2}=$ 0.28). Concerning the case of IH, we find that values of $\theta_{23}$ in the first octant are allowed only with $\Delta \chi^{2}>1.9$, to be compared with $\Delta \chi^{2}>1.5$ before the inclusion of new reactor data.

The new determination of $\theta_{13}$ at reactor experiments also has an impact on the sensitivity to the $C P$ phase $\delta$ from the global oscillation analysis. The lower value implied by new data increases the tension between long-baseline and reactor data for some values of $\delta$, enhancing the rejection against $\delta \sim 0.5 \pi$. We find that these values are now disfavored at the $2.0 \sigma(2.7 \sigma)$ level for normal (inverted) mass ordering.

\section{B. Summary of the updated global neutrino oscillation analysis}

The main results of our updated global fit to neutrino oscillations are summarized in Fig. 5 and Table [1 As commented above, the new reactor data presented at the Neutrino 2014 conference show a preference for a lower 

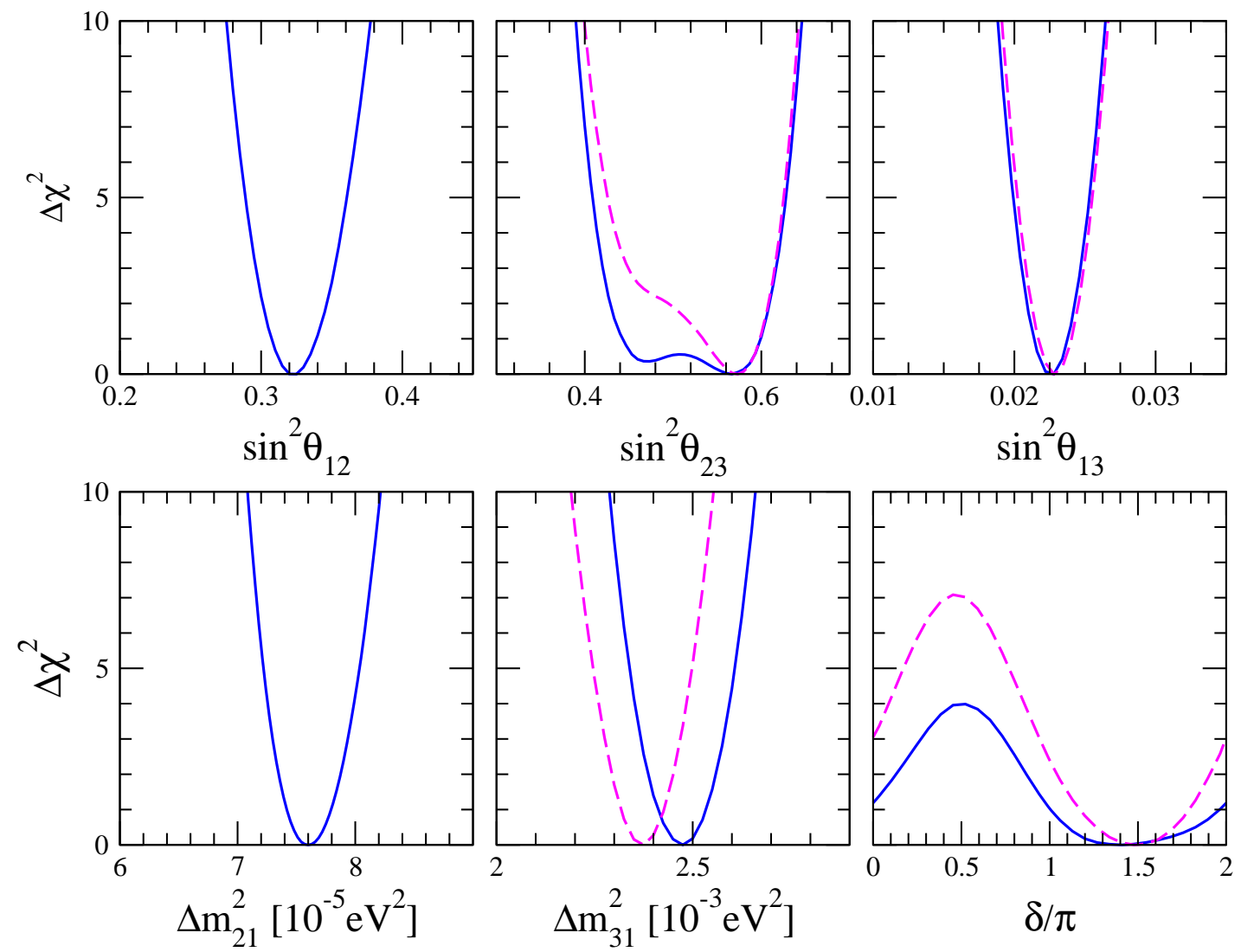

FIG. 5: Same as Fig. 4 for the updated global analysis after Neutrino 2014 conference

value of $\sin ^{2} \theta_{13}$, now determined with an accuracy of $\sim 5 \%$ thanks to the more precise data. This modification leaves unchanged all the other oscillation parameters except the atmospheric mixing angle $\theta_{23}$ and the $C P$ phase $\delta$. In both cases, the smaller value of $\theta_{13}$ favored by the new data results in a worsening of the solutions already disfavored in our previous analysis, namely, first octant solutions for $\theta_{23}$ and values of $\delta \sim 0.5 \pi$. 


\begin{tabular}{|c|c|c|c|}
\hline parameter & best fit $\pm 1 \sigma$ & $2 \sigma$ range & $3 \sigma$ range \\
\hline$\Delta m_{21}^{2}\left[10^{-5} \mathrm{eV}^{2}\right]$ & $7.60_{-0.18}^{+0.19}$ & $7.26-7.99$ & $7.11-8.18$ \\
\hline$\left|\Delta m_{31}^{2}\right|\left[10^{-3} \mathrm{eV}^{2}\right](\mathrm{NH})$ & $2.48_{-0.07}^{+0.05}$ & $2.35-2.59$ & $2.30-2.65$ \\
\hline$\left|\Delta m_{31}^{2}\right|\left[10^{-3} \mathrm{eV}^{2}\right](\mathrm{IH})$ & $2.38_{-0.06}^{+0.05}$ & $2.26-2.48$ & $2.20-2.54$ \\
\hline $\sin ^{2} \theta_{12} / 10^{-1}$ & $3.23 \pm 0.16$ & $2.92-3.57$ & $2.78-3.75$ \\
\hline$\theta_{12} /^{\circ}$ & $34.6 \pm 1.0$ & $32.7-36.7$ & $31.8-37.8$ \\
\hline $\sin ^{2} \theta_{23} / 10^{-1}(\mathrm{NH})$ & $5.67_{-1.24}^{+0.32 a}$ & $4.14-6.23$ & $3.93-6.43$ \\
\hline$\theta_{23} /^{\circ}$ & $48.9_{-7.2}^{+1.8}$ & $40.0-52.1$ & $38.8-53.3$ \\
\hline $\sin ^{2} \theta_{23} / 10^{-1}(\mathrm{IH})$ & $5.73_{-0.39}^{+0.25}$ & $4.35-6.21$ & $4.03-6.40$ \\
\hline$\theta_{23} /^{\circ}$ & $49.2_{-2.3}^{+1.5}$ & $41.3-52.0$ & $39.4-53.1$ \\
\hline $\sin ^{2} \theta_{13} / 10^{-2}(\mathrm{NH})$ & $2.26 \pm 0.12$ & $2.02-2.50$ & $1.90-2.62$ \\
\hline$\theta_{13} /^{\circ}$ & $8.6_{-0.2}^{+0.3}$ & $8.2-9.1$ & $7.9-9.3$ \\
\hline $\sin ^{2} \theta_{13} / 10^{-2}(\mathrm{IH})$ & $2.29 \pm 0.12$ & $2.05-2.52$ & $1.93-2.65$ \\
\hline$\theta_{13} /^{\circ}$ & $8.7 \pm 0.2$ & $8.2-9.1$ & $8.0-9.4$ \\
\hline$\delta / \pi(\mathrm{NH})$ & $1.41_{-0.40}^{+0.55}$ & $0.0-2.0$ & $0.0-2.0$ \\
\hline$\delta /^{\circ}$ & $254_{-72}^{+99}$ & $0-360$ & $0-360$ \\
\hline$\delta / \pi(\mathrm{IH})$ & $1.48 \pm 0.31$ & $0.00-0.09 \& 0.86-2.0$ & $0.0-2.0$ \\
\hline$\delta /{ }^{\circ}$ & $266 \pm 56$ & $0-16 \& 155-360$ & $0-360$ \\
\hline
\end{tabular}

${ }^{a}$ There is a local minimum in the first octant, at $\sin ^{2} \theta_{23}=0.473$ with $\Delta \chi^{2}=0.36$ with respect to the global minimum

TABLE II: Neutrino oscillation parameters summary from the global analysis updated after Neutrino 2014 conference

[1] J. Schechter and J. W. F. Valle, Phys. Rev. D22, 2227 (1980).

[2] J. Beringer et al. (Particle Data Group), Phys.Rev. D86, 010001 (2012).

[3] W. Rodejohann and J. Valle, Phys.Rev. D84, 073011 (2011), 1108.3484.

[4] F. An et al. (DAYA-BAY Collaboration), Phys.Rev.Lett. 108, 171803 (2012), 1203.1669.

[5] J. Ahn et al. (RENO collaboration), Phys.Rev.Lett. 108, 191802 (2012), 1204.0626.

[6] M. Apollonio et al. (CHOOZ), Eur. Phys. J. C27, 331 (2003), hep-ex/0301017.

[7] F. Boehm et al. (Palo Verde collaboration), Phys. Rev. D64, 112001 (2001), hep-ex/0107009.

[8] K. Abe et al. (T2K Collaboration), Phys.Rev.Lett. 107, 041801 (2011), 1106.2822.

[9] P. Adamson et al. (MINOS Collaboration), Phys.Rev.Lett. 107, 181802 (2011), 1108.0015.

[10] D. Forero, M. Tortola, and J. W. F. Valle, Phys.Rev. D86, 073012 (2012), arXiv:1205.4018.

[11] F. An et al. (Daya Bay Collaboration) (2013), 1310.6732.

[12] J. Ling's talk at TAUP 2013 conference, Asilomar. (2013), URL https://conferences.lbl.gov/contributionDisplay $\cdot$ py?sessionId=36\&contribId=75\&conf Id=36.

[13] S. Seon-Hee's talk at TAUP 2013 conference, Asilomar. (2013), URL https://conferences. Ibl.gov/contributionDisplay . py?sessionId=36\&contribId=121\&conf Id=36

[14] P. Adamson et al. (MINOS Collaboration), Phys.Rev.Lett. 110, 251801 (2013), 1304.6335.

[15] P. Adamson et al. (MINOS Collaboration), Phys.Rev.Lett. 110, 171801 (2013), 1301.4581.

[16] K. Abe et al. (T2K Collaboration), Phys.Rev.Lett. 112, 181801 (2014), 1403.1532.

[17] K. Abe et al. (T2K Collaboration) (2013), 1311.4750.

[18] K. Abe et al. (Super-Kamiokande Collaboration), Phys.Rev. D83, 052010 (2011), 1010.0118.

[19] A. Renshaw (Super-Kamiokande Collaboration) (2014), 1403.4575. 
[20] B. T. Cleveland et al., Astrophys. J. 496, 505 (1998).

[21] F. Kaether, W. Hampel, G. Heusser, J. Kiko, and T. Kirsten, Phys.Lett. B685, 47 (2010), 1001.2731.

[22] J. N. Abdurashitov et al. (SAGE Collaboration), Phys. Rev. C80, 015807 (2009), 0901.2200.

[23] G. Bellini, J. Benziger, D. Bick, S. Bonetti, G. Bonfini, et al., Phys.Rev.Lett. 107, 141302 (2011), 1104.1816.

[24] B. Aharmim et al. (SNO), Phys. Rev. Lett. 101, 111301 (2008), 0806.0989.

[25] B. Aharmim et al. (SNO), Phys. Rev. C81, 055504 (2010), 0910.2984.

[26] J. Hosaka et al. (Super-Kamkiokande), Phys. Rev. D73, 112001 (2006), hep-ex/0508053.

[27] J. Cravens et al. (Super-Kamiokande Collaboration), Phys.Rev. D78, 032002 (2008), 0803.4312.

[28] T. Schwetz, M. Tortola, and J. W. F. Valle, New J. Phys. 13, 063004 (2011).

[29] T. Schwetz, M. Tortola, and J. Valle, New J.Phys. 13, 109401 (2011), 1108.1376.

[30] F. An et al. (Daya Bay Collaboration), Chin.Phys. C37, 011001 (2013), 1210.6327.

[31] Y. Abe et al. (Double Chooz Collaboration), Phys.Rev. D86, 052008 (2012), 1207.6632.

[32] P. Huber, J. Kopp, M. Lindner, M. Rolinec, and W. Winter, Comput.Phys.Commun. 177, 432 (2007), hep-ph/0701187.

[33] K. Abe et al. (T2K Collaboration), Phys.Rev.Lett. 111, 211803 (2013), 1308.0465.

[34] A. Gando et al. (The KamLAND Collaboration), Phys.Rev. D83, 052002 (2011), 1009.4771.

[35] R. Wendell et al. (Super-Kamiokande Collaboration), Phys.Rev. D81, 092004 (2010), 1002.3471.

[36] Y. Itow, Nucl.Phys.Proc.Suppl. 235-236, 79 (2013).

[37] A. Himmel (Collaboration for the Super-Kamiokande) (2013), 1310.6677.

[38] M. Gonzalez-Garcia, M. Maltoni, J. Salvado, and T. Schwetz, JHEP 1212, 123 (2012), 1209.3023. URL www.nu-fit.org.

[39] F. Capozzi, G. L. Fogli, E. Lisi, A. Marrone, D. Montanino and A. Palazzo, Phys. Rev. D 89, 093018 (2014), 1312.2878.

[40] Y. Abe et al. [Double Chooz Collaboration], arXiv:1406.7763 [hep-ex].

[41] H. de Kerret's talk at Neutrino 2014 conference, Boston.

[42] Seon-Hee Seo's talk at Neutrino 2014 conference, Boston.

[43] Chao Zhang's talk at Neutrino 2014 conference, Boston.

[44] Weili Zhong's talk at the ICHEP 2014 conference, Valencia. 